\title{
An Analysis of Typically Developing Turkish Kindergarten Children's Attitudes toward Their Peers with Special Needs
}

\author{
Mümin TUFAN, Elizabeth Blue SWADENER
}

Summary

\section{PURPOSE}

Because of the observational learning, societal influences, and the environment in which they are raised, several children enter school holding misconception and stereotypical views about persons they perceive as different.

Because negative attitudes are extremely effective in limiting opportunities for children with special needs, a better understanding of young children's ideas about their peers with special needs to diminish this negative effect may be extremely crucial.

It is important to consider that even though young children are aware of and curious about differences, they may not have set ideas and attitudes toward these differences. This point of view leads to the possibility that attitudes toward special needs may be positively influenced by the integration experience.

Therefore, understanding of children's beliefs about, knowledge of and attitudes toward their peers with individual differences and attitudes is essential for the enhancement of relationships between these two groups.

\section{METHOD}

This exploratory study employed a qualitative design, using participant observations and semi-structured conversational interviews to gather data in an effort to provide a rich portrait of events, experiences, and perspectives pertinent to cross-ability interactions in Turkish kindergarten classrooms. Qualitative analysis of semi-structured interviews with open-ended questions and field observations were used to gain more insight related with children's attitudes toward their classmates with special needs with the support of field observations.

Total of 10 children, eight typically developing and two with special needs in two inclusive kindergarten classrooms, their parents, and their classroom teachers were participated in this study. All children were between 5 and 6 years of age and participants were given names which were all pseudonyms.

During interviews the answers were carefully written down and occurrences during field observations taken notes by the researcher. The data was analyzed carefully by using qualitative content analysis method and the themes were constructed.

\section{RESULTS AND DISCUSSION}

Consistent with previous findings kindergarten-age children in this study also demonstrated that they can notice and have some degree of knowledge about the differences of people with special needs around them. After learning about typically developing children's knowledge about disabilities and attitude-related themes, based on an analysis of both observations and interview data, were presented. These themes were categorized into two broad categories, according to being positive or negative framings. Positive topics mostly included responses of typically developing children regarding liking their peers with special needs, helping and protecting them, and involving them into their play. Negative topics generally appeared in the observations that focused on the typically developing children's low 
expectations regarding their peers with special needs, ignoring and rejecting them, and playing rough with them.

Based on the responses of typically developing children to the interview questions and some of their observed interactions, participant children in this study were mostly accepting of individuals with special needs.

Even though children's interview responses did not directly indicate their hesitance in becoming friends or playing with a child with special needs, during interviews and their observed behaviors showed their tendency in some cases to underestimate their peers' potential.

The attitudes and the interaction patterns were not always positive. Children's answers to the interview questions and their actual interaction patterns sometimes varied.

\section{Implications for Practice}

An extended study might include a larger sample of children from different socioeconomic, environmental, and culturally diverse backgrounds.

According to teachers' responses, there was no set curriculum that focused on promoting acceptance of children with special needs by their peers with typical development. This can be addressed in the future studies that the aim may be on constructing a curriculum, training the teachers, and putting it into practice. Different ideas, different curriculums, different environments and applications may provide positive results.

Following research may be conducted in smaller cities and comparisons can be made between schools, classroom, with children from different socioeconomic families, different age groups, genders, and apparent and less apparent disabilities.

The future research should be focusing on the reasons of the differences between children's statements and their actual actions. 